\title{
Reliability, maintainability and sensitivity analysis of physical processing unit of sewage treatment plant
}

\author{
Drishty Goyal ${ }^{1} \cdot$ Ashish Kumar $^{1} \cdot$ Monika Saini $^{1} \cdot$ Hemlata Joshi ${ }^{2}$
}

Received: 5 August 2019 / Accepted: 18 October 2019 / Published online: 29 October 2019

(c) Springer Nature Switzerland AG 2019

\begin{abstract}
India is facing radical change in perspective of inadequate water resources which can reduce by using treated water. In this direction, sewage treatment plants play a key role. Sewage treatment plant comprises three units namely physical processing, chemical processing and biological process. The physical process is the most important part and it has five component arranged in series configuration. It becomes necessary to perform this process with high efficiency and reliability, availability, maintainability, and dependability (RAMD) is the methodology to analyze the performance. The failure and repair rates of the subsystems has been considered exponentially distributed. Chapman-Kolmogorov differential equations are derived using Markovian birth-death process and several measures like mean time between failures, mean time to repair and dependability ratio are derived. The sensitivity analysis of reliability of the plant has also been performed. RAMD investigation shows that: availability of system is 0.952177 , reliability of the system after 20 days is 0.2018 and after 60 days 0.00823 , maintainability of the plant is 0.999948 , dependability ratio is 0.9541 and raw sewage sump is the most sensitive subsystem of the plant with reliability 0.382893 . This work is projected to support as an informative exertion in steering a RAMD analysis of physical processing unit and vary few work is available in literature related to the performance features of physical processing units of the sewage treatment plants. The main findings may be very useful for sewage treatment plants designers.
\end{abstract}

Keywords Physical processing unit $\cdot$ Availability $\cdot$ Reliability $\cdot$ Maintainability $\cdot$ Dependability ratio

Sist of symbols
Cystem is working with full capacity
Failure rate of subsystems $\mathrm{R}$ and $\mathrm{R}_{1}$ Failure rate of subsystems $S$ Failure rate of subsystems $T$ Failure rate of subsystems $S_{1}$ Repair rate of subsystems $P$ Repair rate of subsystems $Q$ Repair rate of subsystems $R$ and $R_{1}$ Repair rate of subsystems $S$ Repair rate of subsystems $T$ Repair rate of subsystems $S_{1}$ Probability that system is in initial state with full capacity

Ashish Kumar, ashishbarak2020@gmail.com | 'Department of Mathematics and Statistics, School of Basic Science, Manipal University, Jaipur, Jaipur 303007, India. ${ }^{2}$ Department of Statistics, CHRIST University, Bangalore, India. 


\section{$P_{j}(t), j=1,2,3$ Probability that subsystem is at the $j$ th state at time $t$ \\ $P_{j}^{\prime}(t), j=1,2,3 \quad$ Represent the derivative of the equa- tions with respect to time $t$}

\section{Introduction}

Since the last few decades, humans have been trying to identify the effective way for reducing the inadequate water problems. Reuse of water after treatment is an effective way to sort out this issue. Sewage treatment plant (STP) can be used for recycling the used water as a fruitful mechanism in this drastic situation. In this scenario, it becomes necessary that STP performs with full efficiency that can be achieved only if each and every component of the system used in STP work with high reliability and proper maintenance strategies are performed. It is a well-known fact that every industry is established with a motto either profit making or social welfare. And, it can be achieved by increasing the productivity of the industry that can be possible only by using reliable components and proper maintenance policy scheduling. That's why the maintenance team of every industry looks for adequate method for adapting operation maintenance strategy. Reliability, availability, maintainability and dependability (RAMD) plays a very important role in recognition of critical components and establishment of maintenance strategies. On the basis of maintenance strategies, reliability, availability and mean time between failures (MTBF) can be exaggerated. By examining reliability, availability and dependability the failure rate of all the subsystems can be reduced by conducting the maintenance of the system. By using RAMD analysis, some measures of subsystems like reliability, dependency ratio, availability, maintainability, mean time between failures, mean time to repair can be obtained.

In existing literature, researchers have been applied various methods to analyze reliability measures. Ebeling [1] proposed some reliability and maintainability policies, explained techniques for failure and repair rate analysis and testing the reliability of data. Komal and Kumar [2] suggested a method for the estimation of RAM indices by using traditional lambda-tau methodology. Garg and Sharma [3] developed a two phase approach namely particle swarm optimization (PSO) to establish the concept of moment method for identify the distribution parameters with the help of mean, standard deviation, and coefficient of variation for failure data. Aggarwal et al. [4] suggested a methodology to measure RAMD indices for skim milk powder production system in a dairy plant using markov birth-death process and calculated RAMD indices for this system. Addabo et al. [5] analyzed reliability and availability of multicore control system of a UPS modular system. De Sanctis et al. [6] proposed a method to increase the performance of industry and suggested engineers some maintenance strategies to manage problems of high cost, safety and environment protection. For this, RAMD analysis has been done by considering oil gas sector equipment's as a case study object. Aggarwal et al. [7] presented a methodology for identification of complex components in refining system of sugar plant and developed Chapman-Kolmogorov differential equation using Markov birth-death process. The numerical results of MTBF, MTTR and dependency ratio have been derived for the system. Corvaro et al. [8] studied the behavior and performance of the component, in reciprocating compressors and identified the critical components to improve reliability of the systems. And, calculate reliability, availability and maintainability of the system. Tsarouhas [9-14] analyzed statistically analyzed reliability, availability and maintainability of many industrial systems like wine packaging system, cheese production plant. The distribution of best-fit has been identified for failure and repairs and maintenance policies are proposed for enhance the efficiency of the plants. Niwas and Garg [15] proposed a method to understand the operational behavior of an industrial system using the concept of warranty of product. Markovian approach is used by assuming constant failure and repair rates of the system. Numerical results for reliability, MTTF, availability and profit have been derived. Choudhary et al. [16] discussed a methodology for improving the reliability of a cement plant. MTBF and MTTR of system calculated over period of 2 years and analyze RAMD indices. Recently, Dahiya et al. [17] and Saini and Kumar [18] used fuzzy reliability and RAMD approaches to analyze performance of A-Pan crystallization system and evaporation system of sugar industry. In the existing literature, no work has been incorporated to analyze the performance of sewage treatment plants. No efforts have been made to improve the performance of physical processing unit of sewage treatment plant. Hence, in present study an effort has been made to analyze the reliability indices of STP. Basic principles of probability theory and Markovian birth-death process has been used to analyze the system. The needful data has been collected from a sewage treatment plant situated in Jaipur with the help of the maintenance personals of the plant. The present study is organized in four sections including the present introductory section. In Sect. 2, various useful definitions and system description, notations are appended. RAMD analysis in performed in 
Sect. 3 while Sect. 4 is devoted to conclusion and implications of the results.

\section{Materials and methods}

\subsection{System description}

In this study, all the measures are derived on component wise. The failure and repair rates of all the subsystems are consider as exponentially distributed. In this section, a brief description of physical processing unit of sewage treatment plant has been given. Physical processing unit consists of five subsystems namely inlet channel, screen chamber, raw sewage sump, fine screen and grit chamber. All subsystems are arranged in series configuration whereas the components also have some internal redundancy. The pictorial representation of system is shown with a flow chart in Fig. 1. The detailed description is as follows:

\section{Subsystem 'A' (inlet channel)}

It consist only one unit of inlet channel in series configure with other subsystem screen channel. Complete system can fail due to failure of it. In this subsystem, all sewerage water collect and then transfer towards screen chamber.

\section{Subsystem 'B' (screen chamber)}

It consist only one unit of screen chamber in series configure with other subsystem inlet channel and raw sewage sump. Complete system can fail due to failure in this subsystem.

\section{Subsystem ' $C$ ' (raw sewage sump)}

It consist four unit of raw sewage sump configure as 3-out-of-3: G system with one standby unit and this complete subsystem configure with other subsystem screen chamber and fine screen. Failure of more than two units can cause a complete failure of system. In this subsystem, raw sewage water transfer to the fine screen with the help of vacuum pressure.

\section{Subsystem 'D' (fine screen)}

It consist two unit one is manual and one is mechanical configure as one is operative and one is standby unit and this complete subsystem configure with other subsystem fine screen and grit chamber. Failure rate these subsystems are different from each other. Failure in both unit can cause in failure of complete system. In this subsystem, solid waste removed completely.

\section{Subsystem ' $E^{\prime}$ (grit chamber)}

It consist two unit of grit chamber configure as 2-out-of-2: $\mathrm{G}$ system and this complete subsystem configure with subsystem fine screen. Failure rate of both the components in subsystems is same. Failure of both unit can cause in a complete system failure. In this subsystem, small waste removed which can't remove by fine screen.

\subsection{RAMD analysis}

In this section, mathematical models of all the subsystems of physical processing unit is formulated and Chapman-Kolmogorov differential equations are derived with

Fig. 1 System description

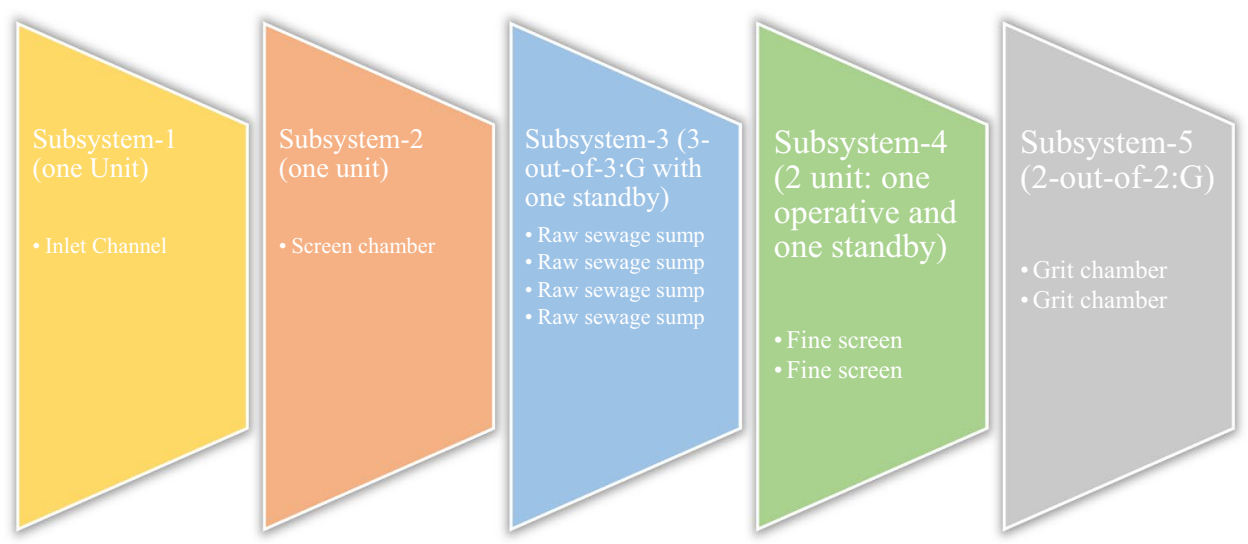


(a)

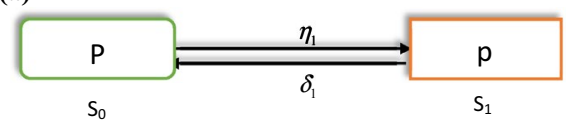

(b)

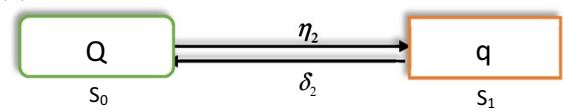

(c)

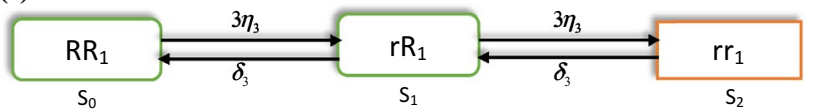

(d)

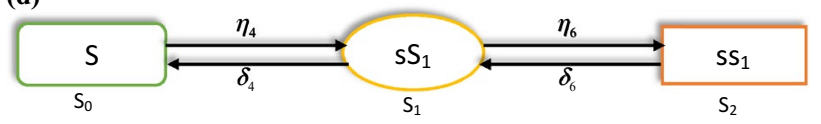

(e)

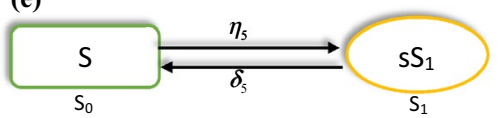

Fig. 2 Transition diagram of a inlet chamber, $\mathbf{b}$ screen chamber, $\mathbf{c}$ raw sewage sump, $\mathbf{d}$ fine screen, e grit chamber

Table 1 In STP failure rate and repair rate of all the subsystems

\begin{tabular}{lll}
\hline Subsystems & Failure rate $(\eta)$ & Repair rate $(\delta)$ \\
\hline$S_{1}$ & Inlet sewer $\left(\eta_{1}\right)=0.003$ & Inlet sewer $\left(\delta_{1}\right)=0.5$ \\
$S_{2}$ & Sluice value $\left(\eta_{2}\right)=0.005$ & Sluice value $\left(\delta_{2}\right)=0.7$ \\
$S_{3}$ & Grit chamber $\left(\eta_{3}\right)=0.008$ & Grit chamber $\left(\delta_{3}\right)=0.9$ \\
$S_{4}$ & Fine screen $\left(\eta_{4}\right)=0.007$ & Fine screen $\left(\delta_{4}\right)=0.9$ \\
& $\left(\eta_{6}\right)=0.005$ & $\left(\delta_{6}\right)=0.7$ \\
$S_{5}$ & Skimming tank & Skimming tank $\left(\delta_{5}\right)=0.6$ \\
& $\left(\eta_{5}\right)=0.006$ & \\
\hline
\end{tabular}

the help of Markov birth-death process. Transition diagrams for all the subsection are shown in Fig. 2a-e and failure and repair rates of all the subsystems are shown in Table 1. The RAMD analysis of subsystems are as follows.

\subsubsection{RAMD indices for subsystem $\mathrm{S}_{1}$}

In subsystem inlet channel, there is only one unit consist in series configuration with other subsystems and failure of it cause the failure of complete system. Differentialdifference equations for the subsystem is derived using birth-death processes on the basis of Fig. 2a. The recurrence relations are as follows:

$P_{0}^{\prime}(t)=-\eta_{1} P_{0}(t)+\delta_{1} P_{1}(t)$

$P_{1}^{\prime}(t)=-\delta_{1} P_{1}(t)+\eta_{1} P_{0}(t)$
With the help of initial conditions and by taking $t \rightarrow \infty$, in Eqs. (1) and (2), we have

$-\eta_{1} P_{0}+\delta_{1} P_{1}=0$

$-\delta_{1} P_{1}+\eta_{1} P_{0}=0$

Now, using normalization condition

$\sum P_{i}=1 ; \quad i=0,1 ; P_{0}+P_{1}=1$

By substituting values of $P_{1}$ derived from Eqs. (3) and (4) in Eq. (5),

Availability of system is as follows:

Availability $=A V_{S_{1}}=\left[1+\frac{\eta_{1}}{\delta_{1}}\right]^{-1}=0.994036$

Reliability of subsystem $\mathrm{S}_{1}$ is derived as follows:

$R_{S_{1}}(t)=e^{-\eta_{1} t}=e^{-0.003 t}$

Maintainability of subsystem $S_{1}$ is derived as follows:

$M_{S_{1}}(t)=1-e^{-\delta_{1} t}=1-e^{-0.5 t}$

Other performance measures of subsystem $\mathrm{S}_{1}$ are as follows:

MTBF $=\frac{1}{\eta_{1}}=333.333333 h ; M T T R=\frac{1}{\delta_{1}}=2 h ; d=\frac{\delta_{1}}{\eta_{1}}=166.666667$

and $D_{\min }\left(S_{1}\right)=1-\left(\frac{1}{d-1}\right)\left(e^{-\frac{\ln d}{d-1}}-e^{-\frac{d \ln d}{d-1}}\right)=0.994183$.

\subsubsection{RAMD indices for subsystem $\mathrm{S}_{2}$}

In subsystem screen chamber only one unit consist in series configuration and failure of it causes the complete system failure. Recurrence relations for this subsystem are derived with the help of Fig. $2 b$ and appended below:

$P_{0}^{\prime}(t)=-\eta_{2} P_{0}(t)+\delta_{2} P_{1}(t)$

$P_{1}^{\prime}(t)=-\delta_{2} P_{1}(t)+\eta_{2} P_{0}(t)$

With the help of initial conditions and by taking $t \rightarrow \infty$, in Eqs. (9) and (10)

$-\eta_{2} P_{0}+\delta_{2} P_{1}=0$

$-\delta_{2} P_{1}+\eta_{2} P_{0}=0$

Now, using normalization condition

$\sum P_{i}=1 ; \quad i=0,1 ; P_{0}+P_{1}=1$

By substituting values of $P_{1}$ derived from Eqs. (11) and (12) in Eq. (13),

Availability of system is derived as follows: 
Availability $=A V_{S_{2}}=\left[1+\frac{\eta_{2}}{\delta_{2}}\right]^{-1}=0.992908$

Reliability of subsystem $\mathrm{S}_{2}$ is obtained as follows:

$R_{S_{2}}(t)=e^{-\eta_{1} t}=e^{-0.005 t}$

Maintainability of subsystem $\mathrm{S}_{2}$ is expressed in the following way:

$M_{S_{2}}(t)=1-e^{-\delta_{1} t}=1-e^{-0.7 t}$

Other performance measures of subsystem $\mathrm{S}_{2}$ are obtained as follows:

MTBF $=\frac{1}{\eta_{2}}=200 h ; M T T R=\frac{1}{\delta_{2}}=1.428571 h ; d=\frac{\delta_{2}}{\eta_{2}}=140$ and $D_{\min }\left(S_{2}\right)=1-\left(\frac{1}{d-1}\right)\left(e^{-\frac{\eta_{2} \ln d}{d-1}}-e^{-\frac{d \ln d}{d-1}}\right)=0.993107$.

\subsubsection{RAMD indices for subsystem $\mathrm{S}_{3}$}

In raw sewage sump subsystem, four unit consist in as 3-out-of-3: G configuration with one standby unit with equal failure and repair rates and failure of two units cause subsystem failure and results the system failure. C-K differential equations for this subsystem are derived using Markovian approach on the basis of state transition diagram appended in Fig. 2c. Recurrence relations are as follows:

$P_{0}^{\prime}(t)=-3 \eta_{3} P_{0}(t)+\delta_{3} P_{1}(t)$

$P_{1}^{\prime}(t)=-\left(3 \eta_{3}+\delta_{3}\right) P_{1}(t)+\delta_{3} P_{2}(t)+3 \eta_{3} P_{0}(t)$

$P_{2}^{\prime}(t)=-\delta_{3} P_{2}(t)+3 \eta_{3} P_{1}(t)$

With the help of initial conditions and by taking $t \rightarrow \infty$, in Eqs. (17-19)

$-3 \eta_{3} P_{0}+\delta_{3} P_{1}=0$

$-\left(3 \eta_{3}+\delta_{3}\right) P_{1}+\delta_{3} P_{2}+3 \eta_{3} P_{0}=0$

$-\delta_{3} P_{2}+3 \eta_{3} P_{1}=0$

Now, using normalization condition

$\sum P_{i}=1 ; \quad i=0,1,2 ; P_{0}+P_{1}+P_{2}=1$

By substituting values of $P_{1}$ and $P_{2}$ derived from Eqs. (20-22) in Eq. (23),

Availability of system is obtained as follows:

Availability $=A V_{S_{3}}=\left(1+\frac{3 \eta_{3}}{\delta_{3}}\right)\left[1+\frac{3 \eta_{3}}{\delta_{3}}+\left(\frac{3 \eta_{3}}{\delta_{3}}\right)^{2}\right]^{-1}$ $=0.999308$

Reliability of subsystem $S_{3}$ is obtained as follows:

$R_{S_{3}}(t)=e^{-3 \eta_{3} t-3 \eta_{3} t}=e^{-0.048 t}$
Maintainability of subsystem $\mathrm{S}_{3}$ as follows:

$M_{S_{3}}(t)=1-e^{-\delta_{3} t-\delta_{3} t}=1-e^{-1.8 t}$

Other performance measures of subsystem $\mathrm{S}_{3}$ are obtained as follows:

$$
\begin{aligned}
& \text { MTBF }=\frac{1}{6 \eta_{3}}=20.833333 h ; M T T R=\frac{M T F(1-A)}{A} \\
= & 0.014430029 h ; d=\frac{M T T F}{M T R}=1443.748542
\end{aligned}
$$

and

$D_{\min }\left(S_{3}\right)=1-\left(\frac{1}{d-1}\right)\left(e^{-\frac{\ln d}{d-1}}-e^{-\frac{d \ln d}{d-1}}\right)=0.999311$.

\subsubsection{RAMD indices for subsystem $\mathrm{S}_{4}$}

In fine screen two unit consists in which one is manual and other mechanical. Here, initially one unit is operative and other kept in cold standby having unequal performance capacity and failure rates and failure of both units causes the complete system failure. $\mathrm{C}-\mathrm{K}$ differential equations for subsystem are derived on the basis of Fig. $2 \mathrm{~d}$. Recurrence relations of system model are expressed as follows:

$P_{0}^{\prime}(t)=-\eta_{4} P_{0}(t)+\delta_{4} P_{1}(t)$

$P_{1}^{\prime}(t)=-\left(\eta_{6}+\delta_{4}\right) P_{1}(t)+\delta_{6} P_{2}(t)+\eta_{4} P_{0}(t)$

$P_{2}^{\prime}(t)=-\delta_{6} P_{2}(t)+\eta_{6} P_{1}(t)$

With the help of initial conditions and by taking $t \rightarrow \infty$, in Eqs. (27-29)

$-\eta_{4} P_{0}+\delta_{4} P_{1}=0$

$-\left(\eta_{6}+\delta_{4}\right) P_{1}+\delta_{6} P_{2}+\eta_{4} P_{0}=0$

$-\delta_{6} P_{2}+\eta_{6} P_{1}=0$

Now, using normalization condition

$\sum P_{i}=1 ; \quad i=0,1,2 ; P_{0}+P_{1}+P_{2}=1$

By substituting values of $P_{1}$ and $P_{2}$ derived from Eqs. (30-32) in Eq. (33),

Availability of system is expressed as follows:

Availability $=A V_{S_{4}}=\left(1+\frac{\eta_{4}}{\delta_{4}}\right)\left[1+\frac{\eta_{4}}{\delta_{4}}+\frac{\eta_{4}}{\delta_{4}} \frac{\eta_{6}}{\delta_{6}}\right]^{-1}=0.9845102$

Reliability of subsystem $\mathrm{S}_{4}$ is obtained as follows:

$R_{S_{4}}(t)=e^{-\eta_{4} t-\eta_{6} t}=e^{-0.012 t}$

Maintainability of subsystem $\mathrm{S}_{4}$ is obtained as follows:

$M_{S_{4}}(t)=1-e^{-\delta_{4} t-\delta_{6} t}=1-e^{-1.6 t}$ 
Other performance measures of subsystem $\mathrm{S}_{4}$ are derived as follows:

$$
\begin{gathered}
\text { MTBF }=\frac{1}{\eta_{4}+\eta_{6}}=83.333333 h ; M T T R \\
=\frac{M T F(1-A)}{A}=1.311124 h ; d=\frac{M T F}{M T T R}=63.558717 \quad \text { and } \\
D_{\min }\left(S_{4}\right)=1-\left(\frac{1}{d-1}\right)\left(e^{-\frac{\ln d}{d-1}}-e^{-\frac{d \ln d}{d-1}}\right)=0.985277 .
\end{gathered}
$$

\subsubsection{RAMD indices for subsystem $\mathrm{S}_{5}$}

The grit chamber consists two units in 2-out-of-2: G configuration and failure rate of both units is same. The failure of one unit causes subsystem failure that results the complete system failure. $\mathrm{C}-\mathrm{K}$ differential equations for this subsystem are derived with the help of Fig. 2e using Markovian birth death process. Recurrence relation are as follows:

$P_{0}^{\prime}(t)=-2 \eta_{5} P_{0}(t)+\delta_{5} P_{1}(t)$

$P_{1}^{\prime}(t)=-\delta_{5} P_{1}(t)+2 \eta_{5} P_{0}(t)$

With the help of initial conditions and by taking $t \rightarrow \infty$, in Eqs. (37) and (38)

$-\eta_{5} P_{0}+\delta_{5} P_{1}=0$
$R_{S_{5}}(t)=e^{-2 \eta_{5} t}=e^{-0.012 t}$

Maintainability of subsystem $\mathrm{S}_{5}$ is derived as follows:

$M_{S_{5}}(t)=1-e^{-\delta_{1} t}=1-e^{-0.6 t}$

Other performance measures of subsystem $\mathrm{S}_{5}$ are obtained as follows:

MTBF $=\frac{1}{\eta_{5}}=83.333333 h ; M T T R=\frac{1}{\delta_{5}}=1.666667 h ; d=\frac{\delta_{5}}{2 \eta_{5}}=50$ and $D_{\min }\left(S_{5}\right)=1-\left(\frac{1}{d-1}\right)\left(e^{-\frac{\ln d}{d-1}}-e^{-\frac{d \ln d}{d-1}}\right)=0.981535$.

\section{Reliability measures}

\subsection{System reliability}

Since the five subsystems comprises the system in series configuration and failure of any one cause the complete failure. The reliability of a series system is equal to the product of the reliability of all the component's reliability. Hence, overall system reliability of physical processing unit is obtained by using Eqs. 7, 15, 25, 35 and 43:

$$
\begin{aligned}
R_{\text {Sys }}(t)=R_{S_{1}}(t) \times R_{S_{2}}(t) \times R_{S_{3}}(t) \times R_{S_{4}}(t) \times R_{S_{5}}(t) & =e^{-\left(\eta_{1}+\eta_{2}+6 \eta_{3}+\eta_{4}+\eta_{6}+2 \eta_{5}\right) t} \\
& =e^{-0.003 t} \times e^{-0.005 t} \times e^{-0.048 t} \times e^{-0.012 t} \times e^{-0.012 t}=e^{-0.08 t}
\end{aligned}
$$

$-\delta_{5} P_{1}+\eta_{5} P_{0}=0$

Now, using normalization condition

$$
\sum P_{i}=1 ; \quad i=0,1 ; P_{0}+P_{1}=1
$$

By substituting values of $P_{1}$ derived from Eqs. (39) and (40) in Eq. (41),

Availability of system obtained as follows:

Availability $=A V_{S_{5}}=\left[1+\frac{2 \eta_{5}}{\delta_{5}}\right]^{-1}=0.980392$

Reliability of subsystem $\mathrm{S}_{5}$ is derived as follows:
Variation in reliability of all the subsystems with respect to time is analyzed by Eq. (45) and appended in Table 2.

\subsection{System availability}

Since all the five subsystems are in series configuration and failure of complete system can occur due to failure of any of these subsystems. The reliability of a series system is equal to the product of the availability of all the component's availability. Hence, overall system availability of physical processing unit is obtained using Eqs. 6, 14, 24, 34 and 42:
Table 2 Variation in reliability of all the subsystems with respect to time

\begin{tabular}{lllllll}
\hline Time (days) & $R_{S_{1}}$ & $R_{S_{2}}$ & $R_{S_{3}}$ & $R_{S_{4}}$ & $R_{S_{5}}$ & $R_{\text {sys }}$ \\
\hline 20 & 0.941765 & 0.904837 & 0.382893 & 0.786628 & 0.786628 & 0.201897 \\
25 & 0.927743 & 0.882497 & 0.301194 & 0.740818 & 0.740818 & 0.135335 \\
30 & 0.913931 & 0.860708 & 0.236928 & 0.697676 & 0.697676 & 0.090718 \\
35 & 0.900325 & 0.839457 & 0.186374 & 0.657047 & 0.657047 & 0.06081 \\
40 & 0.88692 & 0.818731 & 0.146607 & 0.618783 & 0.618783 & 0.040762 \\
45 & 0.873716 & 0.798516 & 0.115325 & 0.582748 & 0.582748 & 0.027324 \\
50 & 0.860708 & 0.778801 & 0.090718 & 0.548812 & 0.548812 & 0.018316 \\
55 & 0.847894 & 0.759572 & 0.071361 & 0.516851 & 0.516851 & 0.012277 \\
60 & 0.83527 & 0.740818 & 0.056135 & 0.486752 & 0.486752 & 0.00823 \\
\hline
\end{tabular}




$$
\begin{aligned}
A_{\text {Sys }}(t)= & A_{S_{1}}(t) \times A_{S_{2}}(t) \times A_{S_{3}}(t) \times A_{S_{4}}(t) \times A_{S_{5}}(t) \\
= & 0.994036 \times 0.993107 \times 0.999308 \\
& \times 0.9845102 \times 0.980392=0.952177
\end{aligned}
$$

\subsection{System maintainability}

Overall maintainability of physical processing unit can be obtained by using Eqs. 8, 16, 26, 36 and 44 in Eq. (47):

$$
\begin{aligned}
M_{\text {Sys }}(t)= & M_{S_{1}}(t) \times M_{S_{2}}(t) \times M_{S_{3}}(t) \times M_{S_{4}}(t) \times M_{S_{5}}(t) \\
= & \left(1-e^{-0.5 t}\right) \times\left(1-e^{-0.7 t}\right) \times\left(1-e^{-1.8 t}\right) \\
& \times\left(1-e^{-1.6 t}\right) \times\left(1-e^{-0.6 t}\right)=1-e^{-0.493213 t}
\end{aligned}
$$

Variation in maintainability of all the subsystems with respect to time is analyzed by the help of Eq. (47) and represented in Table 3.

\subsection{System dependability}

Since all the five subsystems are in series configuration and failure of anyone can cause complete system failure. The dependability of a series system is equal to the product of the dependability of all the component's reliability. Hence, overall system dependability of physical processing unit is as follows:

$$
\begin{aligned}
D_{\min \left(S_{y s}\right)}(t)= & D_{\min \left(s_{1}\right)}(t) \times D_{\min \left(S_{2}\right)}(t) \times D_{\min \left(s_{3}\right)}(t) \\
& \times D_{\min \left(S_{4}\right)}(t) \times D_{\min \left(S_{5}\right)}(t) \\
= & 0.994182 \times 0.993107 \times 0.999311 \\
& \times 0.985277 \times 0.981535=0.954172
\end{aligned}
$$

Summary of all the calculated RAMD indices is appended in Table 4.

\subsection{Sensitivity analysis}

It is a technique which is used to identify the impact of independent variable on a specific dependent variable on the basis of some assign assumptions. It determine the effect of the change in parameters and structure of the model. Here, sensitivity analysis for reliability of the subsystems and system with respect to failure rates $\eta_{v} \eta_{2}, \eta_{3}, \eta_{4}, \eta_{5}$ and $\eta_{6}$ has been performed. The following expressions have been derived respectively:

$\begin{array}{ll}\frac{\partial R_{\text {Sys }}}{\partial \eta_{1}}=-t e^{-\left(\eta_{1}+\eta_{2}+6 \eta_{3}+\eta_{4}+\eta_{6}+2 \eta_{5}\right) t} ; & \frac{\partial R_{\text {Sys }}}{\partial \eta_{2}}=-t e^{-\left(\eta_{1}+\eta_{2}+6 \eta_{3}+\eta_{4}+\eta_{6}+2 \eta_{5}\right) t} ; \\ \frac{\partial R_{\text {Sys }}}{\partial \eta_{3}}=-6 t e^{-\left(\eta_{1}+\eta_{2}+6 \eta_{3}+\eta_{4}+\eta_{6}+2 \eta_{5}\right) t} ; & \frac{\partial R_{\text {Sys }}}{\partial \eta_{4}}=-t e^{-\left(\eta_{1}+\eta_{2}+6 \eta_{3}+\eta_{4}+\eta_{6}+2 \eta_{5}\right) t} ; \\ \frac{\partial R_{\text {Sys }}}{\partial \eta_{5}}=-2 t e^{-\left(\eta_{1}+\eta_{2}+6 \eta_{3}+\eta_{4}+\eta_{6}+2 \eta_{5}\right) t} ; & \frac{\partial R_{\text {Sys }}}{\partial \eta_{6}}=-t e^{-\left(\eta_{1}+\eta_{2}+6 \eta_{3}+\eta_{4}+\eta_{6}+2 \eta_{5}\right) t}\end{array}$

Table 3 Variation in maintainability of all the subsystems with respect to time

\begin{tabular}{lllllll}
\hline Time (days) & $M_{S_{1}}$ & $M_{S_{2}}$ & $M_{S_{3}}$ & $M_{S_{4}}$ & $M_{S_{5}}$ & $M_{\text {sys }}$ \\
\hline 20 & 0.999955 & 0.999999 & 1.000000 & 1.000000 & 0.999994 & 0.999948 \\
25 & 0.999996 & 1.000000 & 1.000000 & 1.000000 & 1.000000 & 0.999996 \\
30 & 1.000000 & 1.000000 & 1.000000 & 1.000000 & 1.000000 & 1.000000 \\
35 & 1.000000 & 1.000000 & 1.000000 & 1.000000 & 1.000000 & 1.000000 \\
40 & 1.000000 & 1.000000 & 1.000000 & 1.000000 & 1.000000 & 1.000000 \\
45 & 1.000000 & 1.000000 & 1.000000 & 1.000000 & 1.000000 & 1.000000 \\
50 & 1.000000 & 1.000000 & 1.000000 & 1.000000 & 1.000000 & 1.000000 \\
55 & 1.000000 & 1.000000 & 1.000000 & 1.000000 & 1.000000 & 1.000000 \\
60 & 1.000000 & 1.000000 & 1.000000 & 1.000000 & 1.000000 & 1.000000 \\
\hline
\end{tabular}

Table 4 RAMD indices for physical processing unit

\begin{tabular}{llllll}
\hline RAMD indices & Subsystem $\left(S_{1}\right)$ & Subsystem $\left(S_{2}\right)$ & Subsystem $\left(S_{3}\right)$ & Subsystem $\left(S_{4}\right)$ & Subsystem $\left(S_{5}\right)$ \\
\hline Reliability & $\mathrm{e}-0.003 \mathrm{t}$ & $\mathrm{e}-0.005 \mathrm{t}$ & $\mathrm{e}-0.048 \mathrm{t}$ & $\mathrm{e}-0.012 \mathrm{t}$ & $\mathrm{e}-0.012 \mathrm{t}$ \\
Maintainability & $1-\mathrm{e}-0.5 \mathrm{t}$ & $1-\mathrm{e}-0.7 \mathrm{t}$ & $1-\mathrm{e}-1.8 \mathrm{t}$ & $1-\mathrm{e}-1.6 \mathrm{t}$ & $1-\mathrm{e}-0.6 \mathrm{t}$ \\
Availability & 0.994036 & 0.992908 & 0.999308 & 0.9845102 & 0.980392 \\
Dependability (d) & 166.666667 & 140 & 1443.748542 & 63.558717 & 50 \\
MTTF & 333.3333332 & 200 & 28.833333 & 83.333333 & 83.333333 \\
MTTR & 2 & 1.428571 & 0.01443003 & 1.311124 & 1.666667 \\
Dep. ratio (Dmin) & 0.994182 & 0.993107 & 0.999311 & 0.985277 & 0.981535 \\
\hline
\end{tabular}




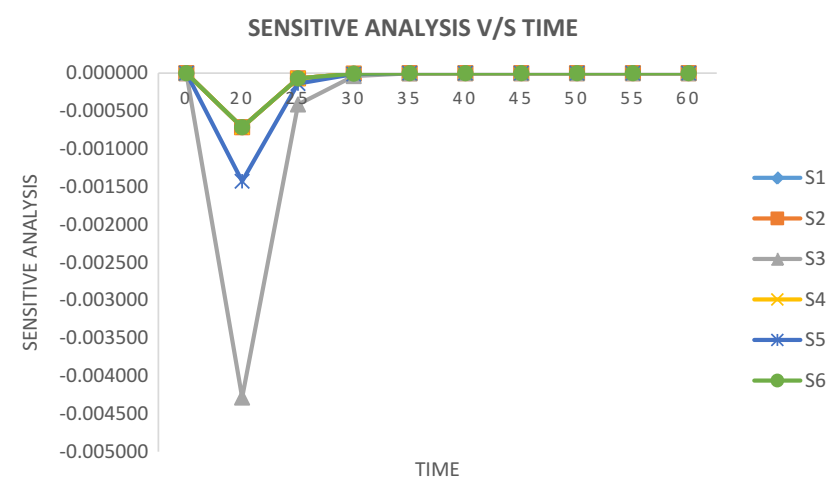

Fig. 3 Sensitivity analysis of system reliability

By putting values of failure rates parameters $\left(\eta_{1}\right)=0.003,\left(\eta_{2}\right)=0.005,\left(\eta_{3}\right)=0.008,\left(\eta_{4}\right)=0.007$, $\left(\eta_{5}\right)=0.006$ and $\left(\eta_{6}\right)=0.005$ in Eq. (49), graphical presentation of sensitive reliability of system is shown in Fig. 3 obtained with respect to time $t=0$ to $t=90$. In Fig. 3, the abbreviations are used as follows:

S1: inlet sewer, S2: sluice value, S3: grit chamber, S4 and S6: fine screen and S5: skimming tank.

\section{Discussion and conclusion}

The reliability analysis of the various subsystems and system has been carried out for a particular case by assigning numerical values appended in Table 1. Reliability and maintainability behavior of various subsystems have been given in Tables 2 and 3. All other RAMD measures are given in Table 4. On the bases of numerical results shown in Table 2, reliability for 30 days is 0.090718 . The reliability behavior of various subsystems with respect to time have been shown in Tables 5, 6, 7, 8 and 9. The probability for system maintenance within 30 days is 0.999996 as shown in Table 3. The reliability of subsystem $S_{3}$ is minimum as compare to reliability of other subsystems hence it need more attention as compare others. From above numerical analysis, it is identified that subsystem $S_{3}$ is highly unreliability and very sensitive with respect to failure rate. Hence subsystem raw sewage sump need high attention to increase the performance of the system. It is concluded that, by controlling the failure rate of raw sewage sump by making proper maintenance strategy the system performance may be increased.

Table 5 Variation in reliability of system due to changes in failure rate of subsystem-1 (inlet channel)

\begin{tabular}{|c|c|c|c|c|c|c|c|c|}
\hline \multirow[t]{2}{*}{ Time (days) } & \multicolumn{4}{|l|}{ System } & \multicolumn{4}{|c|}{ Subsystem-1 } \\
\hline & $\eta_{1}=0.002$ & $\eta_{1}=0.003$ & $\eta_{1}=0.004$ & $\eta_{1}=0.005$ & $\eta_{1}=0.002$ & $\eta_{1}=0.003$ & $\eta_{1}=0.004$ & $\eta_{1}=0.005$ \\
\hline 20 & 0.2059751 & 0.20189652 & 0.1978987 & 0.19398004 & 0.960789 & 0.941765 & 0.923116 & 0.904837 \\
\hline 25 & 0.13876131 & 0.13533528 & 0.13199384 & 0.1287349 & 0.951229 & 0.927743 & 0.904837 & 0.882497 \\
\hline 30 & 0.09348073 & 0.09071795 & 0.08803683 & 0.08543495 & 0.941765 & 0.913931 & 0.88692 & 0.860708 \\
\hline 35 & 0.0629761 & 0.06081006 & 0.05871853 & 0.05669893 & 0.932394 & 0.900325 & 0.869358 & 0.839457 \\
\hline 40 & 0.04242574 & 0.0407622 & 0.0391639 & 0.03762826 & 0.923116 & 0.88692 & 0.852144 & 0.818731 \\
\hline 45 & 0.02858137 & 0.02732372 & 0.02612141 & 0.024972 & 0.913931 & 0.873716 & 0.83527 & 0.798516 \\
\hline 50 & 0.0192547 & 0.01831564 & 0.01742237 & 0.01657268 & 0.904837 & 0.860708 & 0.818731 & 0.778801 \\
\hline 55 & 0.01297151 & 0.01227734 & 0.01162032 & 0.01099846 & 0.895834 & 0.847894 & 0.802519 & 0.759572 \\
\hline 60 & 0.00873865 & 0.00822975 & 0.00775048 & 0.00729913 & 0.88692 & 0.83527 & 0.786628 & 0.740818 \\
\hline
\end{tabular}

Table 6 Variation in reliability of system due to changes in failure rate of subsystem-2 (screen chamber)

\begin{tabular}{|c|c|c|c|c|c|c|c|c|}
\hline \multirow[t]{2}{*}{ Time (days) } & \multicolumn{4}{|l|}{ System } & \multicolumn{4}{|c|}{ Subsystem-2 } \\
\hline & $\eta_{2}=0.004$ & $\eta_{2}=0.005$ & $\eta_{2}=0.006$ & $\eta_{2}=0.007$ & $\eta_{2}=0.004$ & $\eta_{2}=0.005$ & $\eta_{2}=0.006$ & $\eta_{2}=0.007$ \\
\hline 20 & 0.20597533 & 0.20189675 & 0.19789893 & 0.19398026 & 0.923116 & 0.904837 & 0.88692 & 0.869358 \\
\hline 25 & 0.13876106 & 0.13533504 & 0.1319936 & 0.12873467 & 0.904837 & 0.882497 & 0.860708 & 0.839457 \\
\hline 30 & 0.09348072 & 0.09071794 & 0.08803682 & 0.08543494 & 0.88692 & 0.860708 & 0.83527 & 0.810584 \\
\hline 35 & 0.06297618 & 0.06081014 & 0.0587186 & 0.056699 & 0.869358 & 0.839457 & 0.810584 & 0.782705 \\
\hline 40 & 0.04242568 & 0.04076214 & 0.03916384 & 0.0376282 & 0.852144 & 0.818731 & 0.786628 & 0.755784 \\
\hline 45 & 0.02858132 & 0.02732367 & 0.02612136 & 0.02497196 & 0.83527 & 0.798516 & 0.763379 & 0.729789 \\
\hline 50 & 0.01925474 & 0.01831567 & 0.01742241 & 0.01657271 & 0.818731 & 0.778801 & 0.740818 & 0.704688 \\
\hline 55 & 0.01297145 & 0.01227728 & 0.01162026 & 0.01099841 & 0.802519 & 0.759572 & 0.718924 & 0.680451 \\
\hline 60 & 0.00873867 & 0.00822977 & 0.00775051 & 0.00729915 & 0.786628 & 0.740818 & 0.697676 & 0.657047 \\
\hline
\end{tabular}


Table 7 Variation in reliability of system due to changes in failure rate of subsystem-3 (raw sewage sump)

\begin{tabular}{|c|c|c|c|c|c|c|c|c|}
\hline \multirow[t]{2}{*}{ Time (days) } & \multicolumn{4}{|l|}{ System } & \multicolumn{4}{|c|}{ Subsystem-3 } \\
\hline & $\eta_{3}=0.047$ & $\eta_{3}=0.048$ & $\eta_{3}=0.049$ & $\eta_{3}=0.05$ & $\eta_{3}=0.047$ & $\eta_{3}=0.048$ & $\eta_{3}=0.049$ & $\eta_{3}=0.05$ \\
\hline 20 & 0.20597518 & 0.2018966 & 0.19789878 & 0.19398012 & 0.390628 & 0.382893 & 0.375311 & 0.367879 \\
\hline 25 & 0.13876117 & 0.13533515 & 0.13199371 & 0.12873477 & 0.308819 & 0.301194 & 0.293758 & 0.286505 \\
\hline 30 & 0.09348062 & 0.09071785 & 0.08803673 & 0.08543486 & 0.244143 & 0.236928 & 0.229925 & 0.22313 \\
\hline 35 & 0.06297617 & 0.06081013 & 0.05871859 & 0.05669899 & 0.193013 & 0.186374 & 0.179964 & 0.173774 \\
\hline 40 & 0.04242568 & 0.04076214 & 0.03916384 & 0.0376282 & 0.15259 & 0.146607 & 0.140858 & 0.135335 \\
\hline 45 & 0.02858135 & 0.02732369 & 0.02612138 & 0.02497198 & 0.120633 & 0.115325 & 0.110251 & 0.105399 \\
\hline 50 & 0.01925473 & 0.01831567 & 0.0174224 & 0.0165727 & 0.095369 & 0.090718 & 0.086294 & 0.082085 \\
\hline 55 & 0.01297149 & 0.01227733 & 0.01162031 & 0.01099845 & 0.075396 & 0.071361 & 0.067542 & 0.063928 \\
\hline 60 & 0.00873863 & 0.00822973 & 0.00775047 & 0.00729912 & 0.059606 & 0.056135 & 0.052866 & 0.049787 \\
\hline
\end{tabular}

Table 8 Variation in reliability of system due to changes in failure rate of subsystem-4 (fine screen)

\begin{tabular}{|c|c|c|c|c|c|c|c|c|}
\hline \multirow[t]{2}{*}{ Time (days) } & \multicolumn{4}{|l|}{ System } & \multicolumn{4}{|c|}{ Subsystem-4 } \\
\hline & $\eta_{4}=0.011$ & $\eta_{4}=0.012$ & $\eta_{4}=0.013$ & $\eta_{4}=0.014$ & $\eta_{4}=0.011$ & $\eta_{4}=0.012$ & $\eta_{4}=0.013$ & $\eta_{4}=0.014$ \\
\hline 20 & 0.2059752 & 0.20189662 & 0.1978988 & 0.19398014 & 0.802519 & 0.786628 & 0.771052 & 0.755784 \\
\hline 25 & 0.13876112 & 0.13533509 & 0.13199366 & 0.12873472 & 0.759572 & 0.740818 & 0.722527 & 0.704688 \\
\hline 30 & 0.09348076 & 0.09071799 & 0.08803687 & 0.08543498 & 0.718924 & 0.697676 & 0.677057 & 0.657047 \\
\hline 35 & 0.06297616 & 0.06081012 & 0.05871858 & 0.05669898 & 0.680451 & 0.657047 & 0.634448 & 0.612626 \\
\hline 40 & 0.04242572 & 0.04076218 & 0.03916387 & 0.03762824 & 0.644036 & 0.618783 & 0.594521 & 0.571209 \\
\hline 45 & 0.02858133 & 0.02732368 & 0.02612137 & 0.02497196 & 0.609571 & 0.582748 & 0.557106 & 0.532592 \\
\hline 50 & 0.01925473 & 0.01831567 & 0.0174224 & 0.0165727 & 0.57695 & 0.548812 & 0.522046 & 0.496585 \\
\hline 55 & 0.01297145 & 0.01227729 & 0.01162027 & 0.01099841 & 0.546074 & 0.516851 & 0.489192 & 0.463013 \\
\hline 60 & 0.00873867 & 0.00822977 & 0.00775051 & 0.00729915 & 0.516851 & 0.486752 & 0.458406 & 0.431711 \\
\hline
\end{tabular}

Table 9 Variation in reliability of system due to changes in failure rate of subsystem-5 (grit chamber)

\begin{tabular}{|c|c|c|c|c|c|c|c|c|}
\hline \multirow[t]{2}{*}{ Time (days) } & \multicolumn{4}{|l|}{ System } & \multicolumn{4}{|c|}{ Subsystem-5 } \\
\hline & $\eta_{5}=0.011$ & $\eta_{5}=0.012$ & $\eta_{5}=0.013$ & $\eta_{5}=0.014$ & $\eta_{5}=0.011$ & $\eta_{5}=0.012$ & $\eta_{5}=0.013$ & $\eta_{5}=0.014$ \\
\hline 20 & 0.2059752 & 0.20189662 & 0.1978988 & 0.19398014 & 0.802519 & 0.786628 & 0.771052 & 0.755784 \\
\hline 25 & 0.13876112 & 0.13533509 & 0.13199366 & 0.12873472 & 0.759572 & 0.740818 & 0.722527 & 0.704688 \\
\hline 30 & 0.09348076 & 0.09071799 & 0.08803687 & 0.08543498 & 0.718924 & 0.697676 & 0.677057 & 0.657047 \\
\hline 35 & 0.06297616 & 0.06081012 & 0.05871858 & 0.05669898 & 0.680451 & 0.657047 & 0.634448 & 0.612626 \\
\hline 40 & 0.04242572 & 0.04076218 & 0.03916387 & 0.03762824 & 0.644036 & 0.618783 & 0.594521 & 0.571209 \\
\hline 45 & 0.02858133 & 0.02732368 & 0.02612137 & 0.02497196 & 0.609571 & 0.582748 & 0.557106 & 0.532592 \\
\hline 50 & 0.01925473 & 0.01831567 & 0.0174224 & 0.0165727 & 0.57695 & 0.548812 & 0.522046 & 0.496585 \\
\hline 55 & 0.01297145 & 0.01227729 & 0.01162027 & 0.01099841 & 0.546074 & 0.516851 & 0.489192 & 0.463013 \\
\hline 60 & 0.00873867 & 0.00822977 & 0.00775051 & 0.00729915 & 0.516851 & 0.486752 & 0.458406 & 0.431711 \\
\hline
\end{tabular}

\subsection{Decision-making inferences}

Every organization wants to improve their performance and for this they continuously making efforts. For performance analysis management needs simple techniques which does not involve mathematical formulas and calculations. So, RAMD is the approach that helps system managers to analyze the performance effectively:
- It helps in estimation of some measures like mean time between failures (MTBF), mean time to repair (MTTR) and availability for planning proper maintenance strategy.

- Estimation of effect of change in failure and repair rates of the subsystems to increase the performance of the system. 
- In identification of most critical component in the system.

- To determine the nature and distribution of the repair and failure rates.

\section{Compliance with ethical standards}

Conflict of interest On behalf of all authors, I states that there is no conflict of interest.

\section{References}

1. Ebeling C (2008) An introduction to reliability and maintainability engineering, 10th edn. Tata McGraw-Hill, New Delhi

2. Komal SP, Kumar SD (2010) RAM analysis of repairable industrial systems utilizing uncertain data. Appl Soft Comput 10(4):1208-1221

3. Garg H, Sharma SP (2012) A two-phase approach for reliability and maintainability analysis of an industrial system. Int J Reliab Qual Saf Eng 19(3):19

4. Aggarwal A, Kumar S, Singh V (2015) Performance modeling of the skim milk powder production system of a dairy plant using RAMD analysis. Int J Qual Reliab Manag 32(2):167-181

5. Addabo T, Fort A, Mugnaini M, Vignoli V, Simoni E, Mancini M (2016) Availability and reliability modeling of multicore controlled UPS for datacenter applications. Reliab Eng Syst Saf 149:56-62

6. De Sanctis I, Paciarotti C, Di Giovine O (2016) Integration between RCM and RAM: a case study. Int J Qual Reliab Manag 33(6):852-880

7. Aggarwal AK, Kumar S, Singh V (2017) Performance modeling of the serial processes in refining system of a sugar plant using RAMD analysis. Int J Syst Assur Eng Manag 8(2):1910-1922

8. Corvaro F, Giacchetta G, Marchetti B, Recanati M (2017) Reliability, availability, maintainability (RAM) study on reciprocating compressors. Petroleum 3(2):266-272

9. Tsarouhas P (2018) Reliability, availability and maintainability (RAM) analysis for wine packaging production line. Int J Qual
Reliab Manag 35(3):821-842. https://doi.org/10.1108/IJQRM $-02-2017-0026$

10. Tsarouhas $P(2019)$ Statistical analysis of failure data for estimating reliability, availability and maintainability of an automated croissant production line. J Qual Maint Eng. https://doi. org/10.1108/JQME-04-2018-0029

11. Tsarouhas P, Arvanitoyannis I, Varzakas T (2009) Reliability and maintainability analysis of cheese (feta) production line in a Greek medium-size company: a case study. J Food Eng 94(3-4):233-240

12. Tsarouhas P, Varzakas T, Arvanitoyannis I (2009) Reliability and maintainability analysis of strudel production line with experimental data: a case study. J Food Eng 91(2):250-259

13. Tsarouhas $P$ (2014) Application of statistical approaches for analyzing the reliability and maintainability of food production lines: a case study of mozzarella cheese. In: Granato D, Ares G (eds) Chapter in mathematical and statistical methods in food science and technology. Wiley, Chicago, IL, pp 491-510

14. Tsarouhas $P$ (2015) A practical approach for reliability and maintainability analysis of repairable systems: a case study of polypropylene production for food packaging applications. Int J Reliab Saf 9(4):255-271

15. Niwas R, Garg H (2018) An approach for analyzing the reliability and profit of an industrial system based on the cost free warranty policy. J Braz Soc Mech Sci Eng 40:265. https://doi. org/10.1007/s40430-018-1167-8

16. Choudhary D, Tripathi M, Shankar R (2019) Reliability, availability and maintainability analysis of a cement plant: a case study. Int J Qual Reliab Manag. https://doi.org/10.1108/IJQRM -10-2017-0215

17. Dahiya O, Kumar A, Saini M (2019) Mathematical modeling and performance evaluation of A-Pan crystallization system in a sugar industry. SN Appl Sci. https://doi.org/10.1007/s4245 2-019-0348-0

18. Saini M, Kumar A (2019) Performance analysis of evaporation system in sugar industry using RAMD analysis. J Braz Soc Mech Sci Eng 41:4

Publisher's Note Springer Nature remains neutral with regard to jurisdictional claims in published maps and institutional affiliations. 